LBL-37505

\title{
PROBOSCIS CONTAINER SHAPES FOR THE USML-2 INTERFACE CONFIGURATION EXPERIMENT*
}

\author{
Paul Concus \\ Lawrence Berkeley Laboratory and Department of Mathematics \\ University of California, Berkeley, CA 94720 , U.S.A. \\ Robert Finn \\ Department of Mathematics \\ Stanford University, Stanford, CA 94305, U.S.A. \\ Mark Weislogel \\ Space Experiments Division \\ NASA Lewis Research Center, Cleveland, $\mathrm{OH} 44135$, U.S.A.
}

\author{
Presented at: \\ Ninth European Symposium \\ Gravity Dependent Phenomena in Physical Sciences \\ Berlin, Germany \\ May 2-5, 1995
}

* This work was supported in part by the National Aeronautics and Space Administration under Grant NCC3-329, by the National Science Foundation under Grant. DMS91-069689, and by the Applied Mathematical Sciences subprogram of the Office of Energy Research, U.S. Department of Energy, under Contract Number DE-AC03-76SF00098. 


\section{DISCLAIMER}

This report was prepared as an account of work sponsored by an agency of the United States Government. Neither the United States Government nor any agency thereof, nor any of their employees, make any warranty, express or implied, or assumes any legal liability or responsibility for the accuracy, completeness, or usefuiness of any information, apparatus, product, or process disclosed, or represents that its use would not infringe privately owned rights. Reference herein to any specific commercial product, process, or service by trade name, trademark, manufacturer, or otherwise does not necessarily constitute or imply its endorsement, recommendation, or favoring by the United States Government or any agency thereof. The views and opinions of authors expressed herein do not necessarily state or reflect those of the United States Government or any agency thereof. 


\section{DISCLAIMER}

Portions of this document may be illegible in electronic image products. Images are produced from the best available original document. 


\title{
PROBOSCIS CONTAINER SHAPES FOR THE USML-2 INTERFACE CONFIGURATION EXPERIMENT
}

\author{
Paul Concus \\ Lawrence Berkeley Laboratory and Department of Mathematics \\ University of California, Berkeley, CA 94720, U.S.A. \\ Robert Finn \\ Department of Mathematics \\ Stanford University, Stanford, CA 94305, U.S.A. \\ Mark Weislogel \\ Space Experiments Division \\ NASA Lewis Research Center, Cleveland, OH 44135, U.S.A.
}

\section{Introduction}

Small changes in container shape or in contact angle can give rise to large shifts of liquid in a microgravity environment. Such behavior suggests a means for managing fluids in microgravity and, as one specific possible application, for the accurate determination of contact angle. In connection with this application, we discuss certain containers designed for the forthcoming USML-2 Glovebox Interface Configuration Experiment (ICE) and depict their behavior in preliminary drop tower experiments. The containers are in the form of a circular cylinder with two diametrically opposed "proboscis" protrusions. These shapes are based on the canonical (single) proboscis containers introduced mathematically in [1], which have the properties in the absence of gravity that (i) fluid rises arbitrarily high over the entire proboscis for contact angles less than or equal to a critical value and (ii) the size of the proboscis can be made relatively as large a portion of the container cross section as desired. These properties allow overcoming some of the practical limitations of wedge containers; for the latter too little fluid may participate in the shift at a critical contact angle to be easily observable.

We include below some background material from [2], where computational results for the double proboscis containers are presented.

\section{Governing equations}

Consider a cylindrical container of general cross-section partly filled with liquid, as indicated in Fig. 1. According to the classical theory, an equilibrium interface in the absence of gravity between the liquid and gas (or between two immiscible liquids) is determined by the equations

$$
\begin{aligned}
& \operatorname{div} T u=\frac{1}{R_{\gamma}} \quad \text { in } \Omega, \\
& \nu \cdot T u=\cos \gamma \quad \text { on } \Sigma,
\end{aligned}
$$

where

$$
T u \equiv \frac{\nabla u}{\sqrt{1+|\nabla u|^{2}}}
$$

see, e. g., [3, Chap. 1]. In these equations $\Omega$ is the cross section (base) of the cylindrical container, 


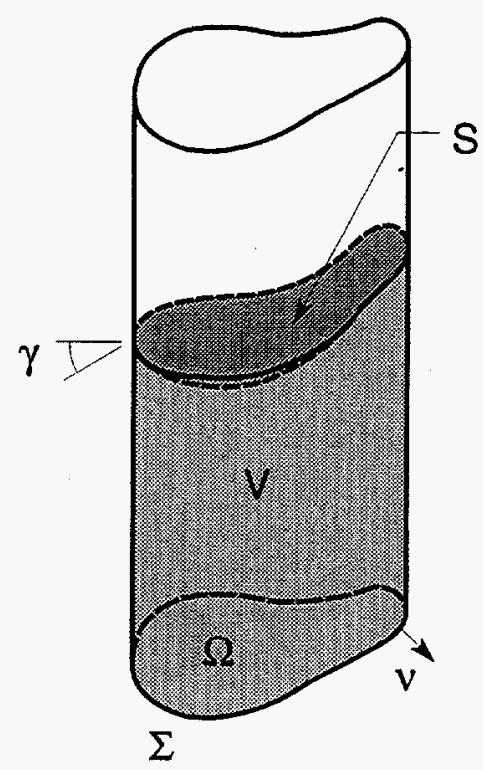

Figure 1. Partly filled cylindrical container with base $\Omega$.

$\Sigma$ is the boundary of $\Omega, \nu$ is the exterior unit normal on $\Sigma$, and

$$
R_{\gamma}=\frac{|\Omega|}{|\Sigma| \cos \gamma}
$$

where $|\Omega|$ and $|\Sigma|$ denote respectively the area and length of $\Omega$ and $\Sigma ; u(x, y)$ denotes the height (single-valued) of the interface $S$ above a reference plane parallel to the base, and $\gamma$ is the contact angle between the interface and the container wall, determined by the material properties. The volume $V$ of liquid in contact with the base is assumed to be sufficient to cover the base entirely, and, for the mathematical results, the cylinder is assumed implicitly to be arbitrarily tall so that questions of behavior at a top do not arise. We restrict discussion to the case of a wetting liquid $0 \leq \gamma<\pi / 2$ (the complementary non-wetting case can be easily transformed into this one). For $\gamma=\pi / 2$, the solution surface is a horizontal plane for any cross-section.

\section{Wedge container}

For a cylindrical container whose section $\Omega$ contains a protruding corner with opening angle $2 \alpha$, as in Fig. 2, the critical value of contact angle, at which behavior is discontinuous, is $\gamma_{0}=\frac{\pi}{2}-\alpha$. For $\frac{\pi}{2}>\gamma \geq \gamma_{0}$ (and for fluid volume sufficient to cover the base) the height $u$ can be given in closed form as the portion of the lower hemisphere with center at $O$ meeting the walls with the prescribed contact angle $\gamma$. Thus the height is bounded uniformly in $\gamma$ throughout this range. For $0 \leq \gamma<\gamma_{0}$, however, the fluid will necessarily move to the corner and rise arbitrarily high at the

vertex, uncovering the base regardless of fluid volume. Details of this behavior can be found in [4], the initial study that revealed the discontinuous behavior, and in [3], [5], and [6]. Procedures for determining contact angle based on the phenomenon can give very good accuracy for larger values of $\gamma$ (closer to $\pi / 2$ ) but may be subject to experimental inaccuracy when $\gamma$ is closer to zero, as the "singular" part of the section over which the fluid accumulates when the critical angle $\gamma_{0}$ is crossed then becomes very small and may be difficult to observe. 


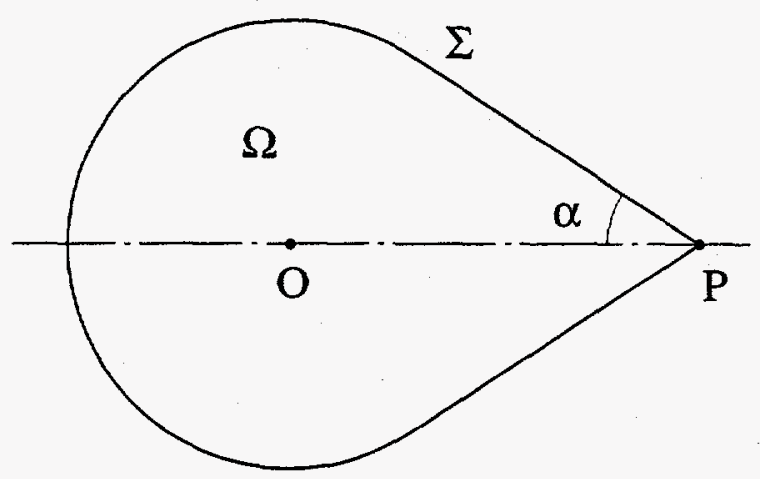

Figure 2. Wedge container section.

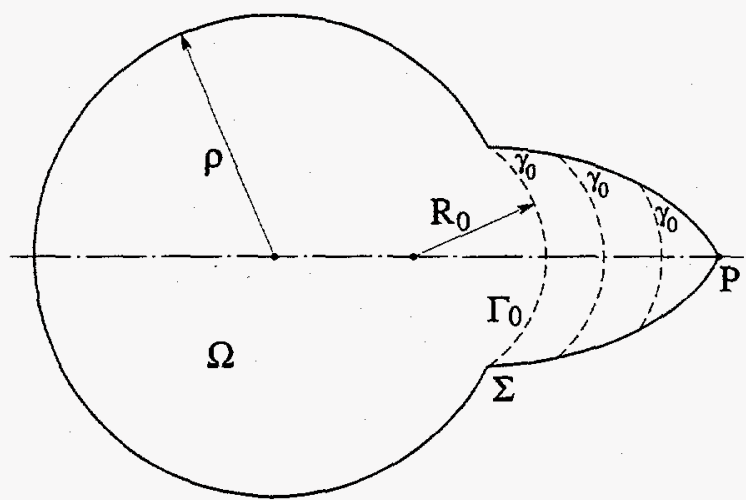

Figure 3. Canonical proboscis container section showing three members of the continuum of extremal arcs.

\section{Canonical proboscis container}

As a way to overcome the experimental difficulty, "canonical proboscis" sections were introduced in [1]. These domains consist of a circular arc attached symmetrically to a (symmetric) pair of curves described by

$$
x=\sqrt{R_{0}{ }^{2}-y^{2}}+R_{0} \sin \gamma_{0} \ln \frac{\sqrt{R_{0}^{2}-y^{2}} \cos \gamma_{0}-y \sin \gamma_{0}}{R_{0}+y \cos \gamma_{0}+\sqrt{R_{0}^{2}-y^{2}} \sin \gamma_{0}}+C,
$$

and meeting at a point $P$ on the $x$-axis, see Fig. 3 . Here $R_{0}$, as well as the particular points of attachment, may be chosen arbitrarily. The (continuum of) circular arcs $\Gamma_{0}$, of which three are depicted by the dashed curves in Fig. 3, are all horizontal translates of one such arc, of radius $R_{0}$ and with center on the $x$-axis, and the curves (4) have the property that they meet all the arcs $\Gamma_{0}$ in the constant angle $\gamma_{0}$. If the radius $\rho$ of the circular boundary arc can be chosen in such a way that $R_{0}$ is the value of $R_{\gamma}$ from (3) for the value $\gamma=\gamma_{0}$, then the arcs $\Gamma_{0}$ become extremals for a "subsidiary" variational problem [7] (see also [3, Chap. 6], [6]) determined by the functional

$$
\Phi \equiv|\Gamma|-\left|\Sigma^{*}\right| \cos \gamma+\left|\Omega^{*}\right| / R_{\gamma}
$$

defined over piecewise smooth arcs $\Gamma$, where $\Sigma^{*}$ and $\Omega^{*}$ are the portions cut off from $\Sigma$ and $\Omega$ by the arcs. In the case of the section of Fig. $3, \Sigma^{*}$ and $\Omega^{*}$ lie to the right of the indicated arcs. It can be shown that every extremal for $\Phi$ is a subarc of a semicircle of radius $R_{0}$, with center on the side 
of $\Gamma$ exterior to $\Omega^{*}$, and that it meets $\Sigma$ in angles $\geq \gamma_{0}$ on the side of $\Gamma$ within $\Omega^{*}$, and $\geq \pi-\gamma_{0}$ on the other side of $\Gamma$ (and thus in angle $\gamma_{0}$ within $\Omega^{*}$ whenever the intersection point is a smooth point of $\Sigma$ ) [7],[3]. It is remarkable that whenever (3) holds, $\Phi=0$ for every $\Omega^{*}$ that is cut off in the proboscis by one of the arcs $\Gamma_{0}$; see [1] and the references cited there.

In [1] a value for $\rho$ was obtained empirically from (3) in a range of configurations, and it was conjectured that the angle $\gamma_{0}$ on which the construction is based would be critical for the geometry. That is, a solution of (1), (2), (3) should exist in $\Omega$ if and only if $\gamma>\gamma_{0}$. Additionally, the fluid height should rise unboundedly as $\gamma$ decreases to $\gamma_{0}$, precisely in the region swept out by the $\operatorname{arcs} \Gamma_{0}$ (the entire proboscis region to the right of the leftmost arc $\Gamma_{0}$ shown in Fig. 3). For these conjectures, which form the basis of our proposed procedure and for which the mathematical underpinnings were proved only partially in [1], complete mathematical proofs have been carried out [8]. Specifically, it has been established that a unique value of $\rho$ can be obtained for any specified proboscis length and that the conjectured behavior of the fluid rise is the only one possible.

In [9] numerical solutions of (1), (2), (3) are depicted for canonical proboscis containers. Although the fluid rise in the corner is not discontinuous as occurs for a planar wedge, it can be "nearly discontinuous" in that the rise height in the proboscis is relatively modest until $\gamma$ decreases to values close to $\gamma_{0}$, and then becomes very rapid at $\gamma=\gamma_{0}$. Furthermore, since the proboscis can be made relatively as large a portion of the section as desired, the shift can be easily observed for a broad range of $\gamma_{0}$. Through proper choice of the domain parameters for the cases considered, an effective balance can be obtained between conflicting requirements for contact angle measurement of a sharp near discontinuity (for accurate measurement) and a sizable volume of fluid rise (for ease of observation).

\section{Double proboscis container}

For the USML-2 experiment, double proboscis containers will be used. These containers are similar to the single proboscis one of Fig. 3, except that there is a second proboscis diametrically opposite to the first, in effect combining two containers into one. The values of $\gamma_{0}$ in (4) generally differ for the left and right proboscides, whose values of $\gamma_{0}$ we denote by $\gamma_{L}$ and $\gamma_{R}$, respectively. Similarly, we denote the values of $R_{0}$ for the left and right proboscides by $R_{L}$ and $R_{R}$. In order for (3) to be satisfied for both proboscides, there holds

$$
R_{R} \cos \gamma_{R}=R_{L} \cos \gamma_{L}
$$

Specifying the desired points of attachment and choosing $\rho$, the radius of the circular portion of the section, so that (3) is satisfied then yields the container section. (Such a $\rho$ can be chosen for the cases discussed here, but a proof that such a choice is possible for any proboscis lengths has not yet been carried out for the double proboscis case.) The critical value for the container is the larger of $\gamma_{L}$ and $\gamma_{R}$. For the containers considered here, we shall take $\gamma_{R}>\gamma_{L}$, so that the critical contact angle $\gamma_{0}$ for the container is equal to $\gamma_{R}$.

The upper half of the sections for the USML-2 experiment, superimposed on one another, are shown in Fig. 4. The sections have been scaled so that the circular portions all have radius unity. The meeting points of the vertices with the $x$-axis are, respectively, a distance 1.5 and 1.6 from the circle center. For the sections depicted in Fig. 4 the values of $\gamma_{L}$ and $\gamma_{R}$ are respectively $20^{\circ}$ and $26^{\circ}$ for the uppermost section, $30^{\circ}$ and $34^{\circ}$ for the middle section, and $38^{\circ}$ and $44^{\circ}$ for the lowest section.

For these containers the explicit behavior has not yet been determined mathematically in complete detail, as it has for the single proboscis containers. However, numerical computations in [2] and the known behavior of the single proboscis solution surfaces suggest that the behavior 


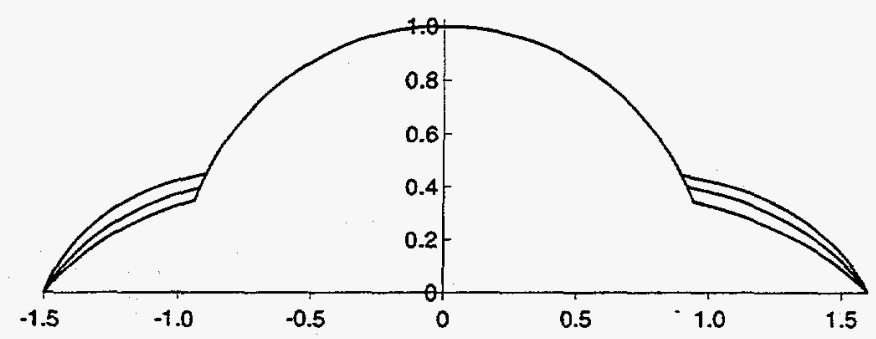

Figure 4. Three superimposed double proboscis container sections. From uppermost to lowest, the pair of values of $\gamma_{0}$ for the left and right proboscides of each section are $20^{\circ} / 26^{\circ}, 30^{\circ} / 34^{\circ}$, and $38^{\circ} / 44^{\circ}$.

will be as follows: For contact angles $\gamma \geq \gamma_{0}$, as $\gamma$ decreases to $\gamma_{0}$ the fluid will rise higher in the right than in the left proboscis, with the rise becoming unbounded in the right proboscis at $\gamma_{0}$. For contact angles between $\gamma_{L}$ and $\gamma_{R}$ the fluid will rise arbitrarily high in the right proboscis, but the height in the left will still be bounded. For smaller contact angles the fluid will rise up both proboscides arbitrarily high. By observing the liquid shift, one can then bracket the contact angle relative to the values of $\gamma_{L}$ and $\gamma_{R}$. For a practical situation in which the container is of finite height with a lid on the top, the fluid will rise to the lid along one or both of the proboscides in the manner described above (providing the fluid volume is adequate).

The selected values of $\gamma_{L}$ and $\gamma_{R}$ for the three containers are based on the value of approximately $32^{\circ}$ measured in a terrestrial environment for the contact angle between the experiment fluid and the acrylic plastic material of the container. The spread of values of contact angle covered by the three containers is intended to allow observation of possible effects of contact angle hysteresis, which is not included in the classical theory.

Typical behavior of the numerical solutions of (1), (2), (3) for the three double proboscis container sections in Fig. 4, for a range of contact angles $\gamma$, is illustrated in Fig. 5, which is taken from [2]. The numerically calculated solution surface $u(x, y)$ for (the upper half of) the $30^{\circ} / 34^{\circ}$ domain is shown for four values of contact angle, $60^{\circ}, 50^{\circ}, 40^{\circ}$, and $35^{\circ}$. (The critical value for the domain is $\gamma_{0}=34^{\circ}$.) Contour levels of the surfaces are indicated by the shading. As for the single proboscis containers, the computations indicate that rise heights are relatively modest until $\gamma$ gets close to the critical value. At the container critical contact angle of $34^{\circ}$, the solution would rise arbitrarily high in the right proboscis. For contact angles less than or equal to $30^{\circ}$, the fluid would rise arbitrarily high in the left as well.

\section{Drop tower tests}

The results of preliminary experiments carried out at the NASA Lewis Zero Gravity Facility 5.18-Sec. drop tower are shown in Fig. 6 for the three vessels of Fig. 4 and for two different liquids. The figure depicts the configurations after approximately five seconds of free fall. In the top row of Fig. 6 , the liquid is a $50 \%$ ethanol solution (the liquid to be used for ICE), for which the equilibrium contact angle with the container wall was measured to be approximately $32^{\circ}$, with a measured receding/advancing hysteresis interval of approximately $18^{\circ}$ to $43^{\circ}$. In the lower row the liquid is a $60 \%$ ethanol solution, for which the equilibrium contact angle is approximately $20^{\circ}$, with a $12^{\circ}-30^{\circ}$ receding/advancing hysteresis interval.

In the approximately five seconds of reorientation from an initial $1-g$ configuration, an indication could be observed of what might occur under the longer-term period of weightlessness of the orbiting Spacelab environment. For the $50 \%$ ethanol mixture, the fluid interfaces rise somewhat along the proboscis portions of the vessel, with a noticeably greater rise in the right proboscis than 


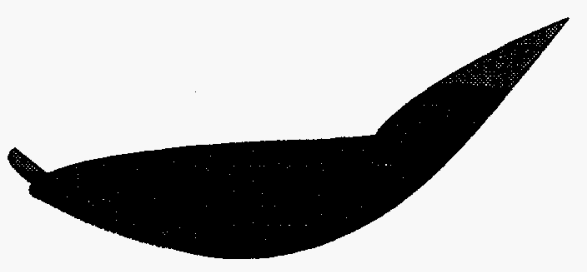

$60^{\circ}$

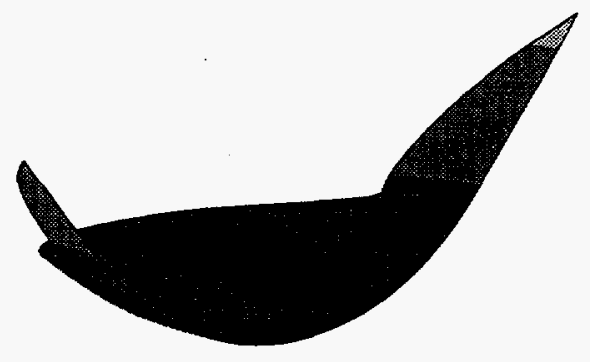

$50^{\circ}$ $40^{\circ}$
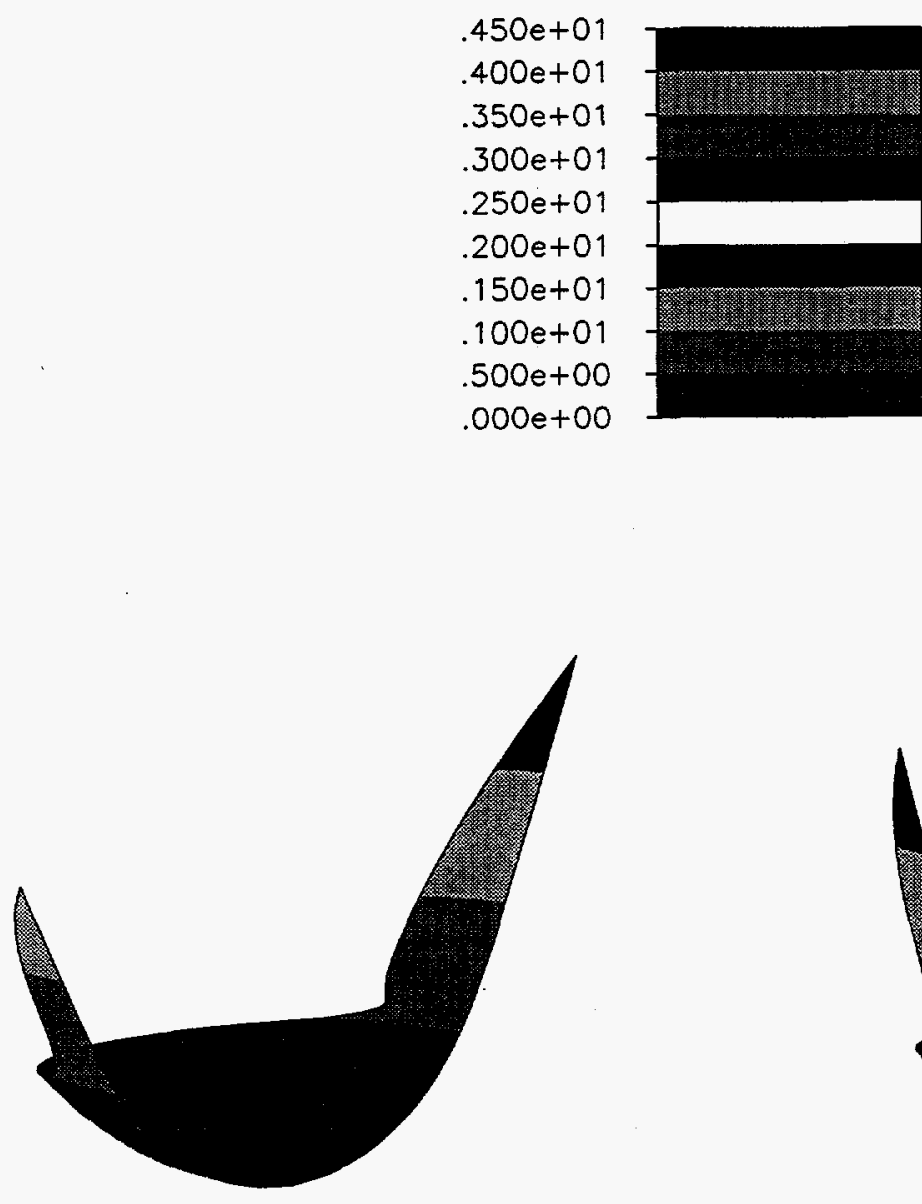

$.000 e+00$

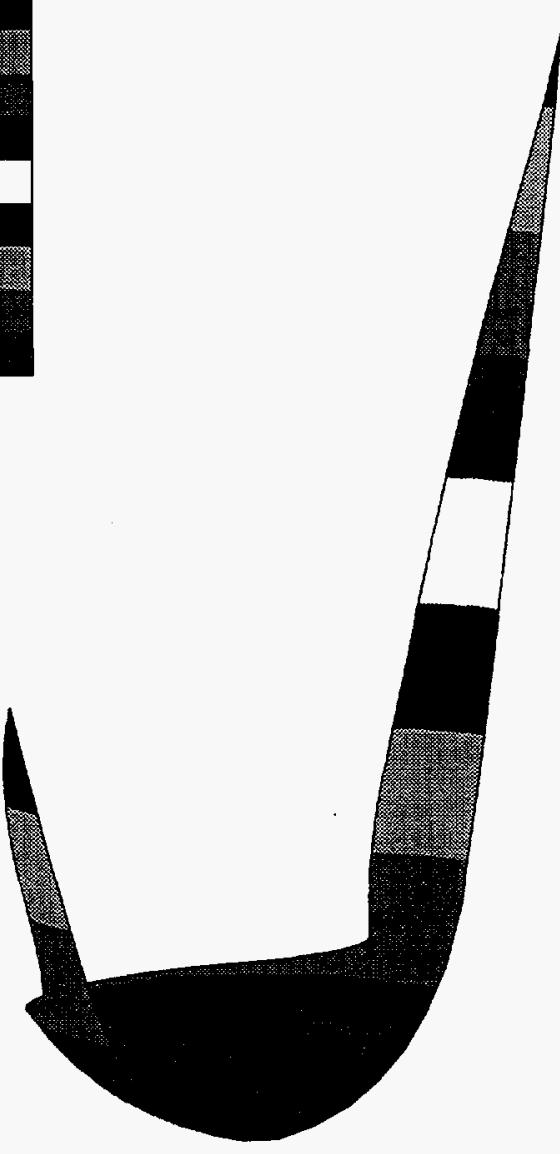

$35^{\circ}$

Figure 5. Equilibrium interface for the $30^{\circ} / 34^{\circ}$ (upper-half) double proboscis section for contact angles $60^{\circ}, 50^{\circ}, 40^{\circ}$, and $35^{\circ}$. $\gamma_{0}=34^{\circ}$. 

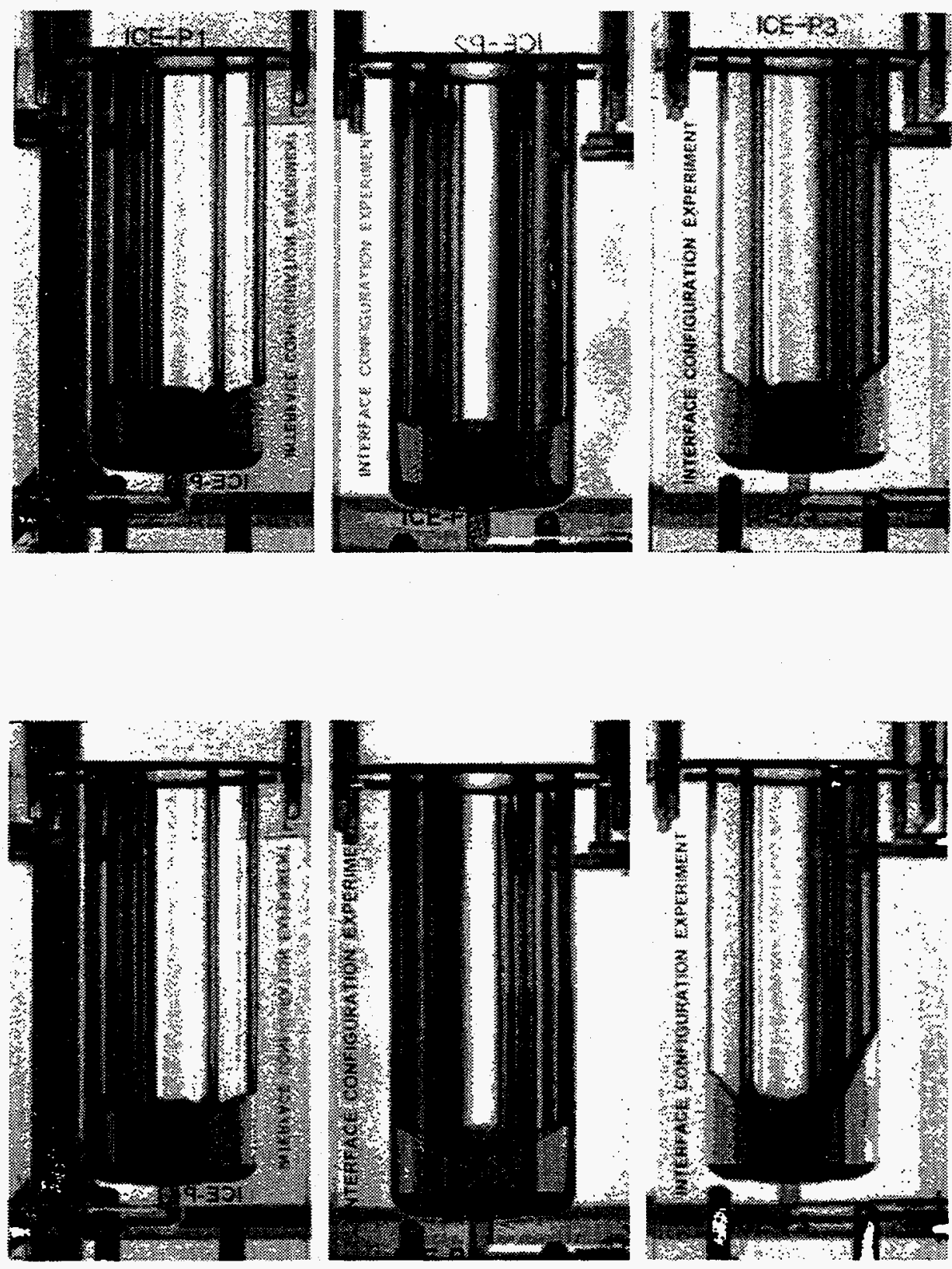

Figure 6. (Transient) drop tower configurations after $\approx 5 \mathrm{sec}$. of free fall. Upper row: $50 \%$ ethanol solution. Lower row: $60 \%$ ethanol solution. From left to right in each row are the $20^{\circ} / 26^{\circ}, 30^{\circ} / 34^{\circ}$, and $38^{\circ} / 44^{\circ}$ vessels, respectively. 
in the left for the $38^{\circ} / 44^{\circ}$ vessel. For the $60 \%$ ethanol mixture the rise is more pronounced; in the $44^{\circ}$ proboscis the substantial rise suggests that the fluid may be proceeding to the top of the container, in accordance with the mathematical results. In the other proboscides the less virgorous rise could be attributed to reorientation forces being smaller when the departure of contact angle from the critical value for the container is less. In a longer term low- $g$ environment and with astronaut "tapping" of the vessels to encourage overcoming of contact line friction and hysteresis effects, we anticipate that information can be obtained as to what extent the mathematical and numerical predictions based on the classical Young-Laplace theory can be observed in practice and what the physical effects might be of factors not included in the classical theory.

\section{ICE experiment}

In addition to the three double proboscis containers depicted in Fig. 4, the USML-2 ICE experiment has also a wedge container. This container is constructed to allow the interior wedge angle $2 \alpha$ (see Fig. 2) to be varied, so as to permit observation of the wedge phenomenon for both the advancing and receding cases.

\section{Acknowledgments}

This work was supported in part by the National Aeronautics and Space Administration under Grant NCC3-329, by the National Science Foundation under Grant DMS91-06968, and by the Mathematical Sciences Subprogram of the Office of Energy Research, U. S. Department of Energy, under Contract Number DE-AC03-76SF00098.

\section{References}

[1] B. Fischer and R. Finn, Non-existence theorems and measurement of capillary contact angle, Zeit. Anal. Anwend. 12 (1993), pp. 405-423.

[2] A. Chen, P. Concus, and R. Finn, On cylinder container sections for a capillary free surface experiment, Paper AIAA 95-0271, 33rd AIAA Aerospace Sciences Meeting, Reno, NV, 1995.

[3] R. Finn, Equilibrium Capillary Surfaces, Springer-Verlag, New York, 1986. Russian translation (with Appendix by H.C. Wente) Mir Publishers, 1988.

[4] P. Concus and R. Finn, On the behavior of a capillary surface in a wedge, Proc. Natl. Acad. Sci., 63 (1969), pp. 292-299.

[5] P. Concus and R. Finn, On capillary free surfaces in the absence of gravity, Acta Math., 132 (1974), pp. 177-198.

[6] P. Concus and R. Finn, Dichotomous behavior of capillary surfaces in zero gravity, Microgravity Sci. Technol. 3 (1990), pp. 87-92; Errata, 3 (1991), p. 230.

[7] R. Finn, A subsidiary variational problem and existence criteria for capillary surfaces, J. reine angew. Math. 353 (1984), pp. 196-214.

[8] R. Finn and T. Leise, On the canonical proboscis, Zeit. Anal. Anwend. 13 (1994), pp. 443-462.

[9] P. Concus, R. Finn, and F. Zabihi, On canonical cylinder sections for accurate determination of contact angle in microgravity, in "Fluid Mechanics Phenomena in Microgravity", AMD Vol. 154, Amer. Soc. Mech. Engineers, D. A. Siginer and M. M. Weislogel, eds., New York, 1992, pp. 125-131. 